\title{
IN VITRO TESTING OF DEFENSE REACTIONS IN ZYGOTIC AND SOMATIC EMBRYOS OF ABIES NUMIDICA ${ }^{1}$
}

\author{
J. Hřib, V. Adamec, B. Vooková \\ Received: April 19, 2011
}

\begin{abstract}
HŘIB, J., ADAMEC, V., VOOKOVÁ, B.: In vitro testing of defense reactions in zygotic and somatic embryos of Abies numidica. Acta univ. agric. et silvic. Mendel. Brun., 2011, LIX, No. 6, pp. 153-160

Defense of desiccated cotyledonary somatic embryos and mature zygotic embryos of Abies numidica was tested in vitro by dual cultures with tester, fungus Phaeolus schweinitzii. Both types of embryos expressed defense reactions manifested by inhibited growth of fungal tester towards the embryos. Mycelial growth was described by logistic sigmoid growth model with a single asymptote. Mutual comparisons of mycelial growth in presence of zygotic and somatic embryos showed significant differences in parameters of mycelium growth curves towards the embryos. Larger defense reactions were observed in zygotic embryos relative to somatic embryos and unlimited control cultivations without embryo. The possible role of auxin in the defense response of plant embryos is discussed.
\end{abstract}

algerian fir, somatic and zygotic embryos, mycelial growth, Phaeolus schweinitzii, dual cultures

There are many reports of previous studies presenting correlations between in vivo and in vitro expression of resistance in trees from experiments with plant tissue cultures (see review by Ostry and Skilling 1992). Use of embryogenic tissue cultures in studies of interactions between Scots pine (Pinus sylvestris L.) and Gremmeniella abietina was reported by Terho et al. (2000).

In our laboratory, defense reactions of conifer zygotic embryos were studied by Hřib et al. (1995ª). The study described defense reactions of zygotic embryos and megagametophyte of Pinus nigra Arn. Basidiomycete Phaeolus schweinitzii was effectively used as a tester for in vitro study of defense reactions. P. nigra seeds presented defense reactions when proliferation of mycelium was inhibited in presence of embryos. From testing of embryogenic and nonembryogenic callus tissue of P. nigra it resulted that the passive defense system in plants had already been formed in early stage of embryogenesis (Hřib et al., 1995'). This conclusion was confirmed by Vooková et al. (2006), who observed defense reactions in developing somatic embryos of A. numidica in presence of $P$. schweinitzii. Both embryonal suspensor mass (ESM) and developing somatic embryos inhibited mycelium growth.

In extension of these experiments, the current study of growth of $P$. schweinitzii mycelium in presence of $A$.numidica zygotic and somatic embryos was conducted. The objective of this study was to evaluate extent of defense reactions in somatic and zygotic embryos relative to untreated control cultivation.

\section{MATERIAL AND METHODS}

Plant material. Zygotic and somatic embryos of Abies numidica de Lannoy ex Carrière were used as an experimental plant material. Zygotic embryos were extirpated from seeds collected from open pollinated trees in Banská Štiavnica, Slovakia. They were sterilized in $70 \%(\mathrm{v} / \mathrm{v})$ ethanol for 30 $\mathrm{s}$ followed by 15 min treatment in $0.1 \%$ (v/v) $\mathrm{HgCl}_{2}$. 
Somatic embryos of A. numidica were obtained from embryogenic cell line No. 12. This embryogenic tissue was induced from immature zygotic embryo, as described by Vooková and Kormuták (2001), and maintained on SH medium (Schenk and Hildebrandt, 1972) with $5 \mu \mathrm{M}$ benzylaminopurine and $1 \%$ sucrose. Maturation of somatic embryos was achieved on modified MS medium (Murashige and Skoog, 1962) supplemented with $4 \%$ maltose, $10 \%$ polyethylene glycol-4000 and $40 \mu \mathrm{M}$ abscisic acid during 8 weeks. Cotyledonary somatic embryos were subjected to partial desiccation during three weeks in the dark at $21-23{ }^{\circ} \mathrm{C}$ (Vooková et al., 2001). Following partial desiccation, somatic embryos with at least four cotyledons were selected in the experiment.

Defense reactions. Defense reactions in mature desiccated cotyledonary somatic and zygotic embryos of $A$. numidica were tested by dual cultures. One somatic or one zygotic embryo were co-cultivated with inoculation of Basidiomycete Phaeolus schweinitzii (Fr.) Pat., which was used as a tester of defense reactions. The tester, P. schweinitzii, isolate No. 151 was obtained from Collection of Wood Destroying Fungi, 1969, Masaryk University in Brno. The culture was grown at a temperature of $24 \pm 1{ }^{\circ} \mathrm{C}$ in the dark on $3 \%$ malt extract and $2 \%$ agar.

The response of $P$. schweinitzii mycelium to placement of the embryos was studied in Petri dishes (Ø $90 \mathrm{~mm}$ ) on agar B-25 medium (Hřib and Rypáček, 1981) without growth regulators. Mature somatic and zygotic embryos were placed on medium at the margin of the dish and cultured in the dark. Inoculation of mycelium was done after 6 days of cultivation. $10 \times 10 \mathrm{~mm}$ pieces of the fungus were taken from the margin of the basic culture on malt agar and placed at a distance of about $2.5 \mathrm{~cm}$ from the embryo. The control dishes were inoculated only with the fungus. The cultures were incubated at $23 \pm 1{ }^{\circ} \mathrm{C}$ in the dark. The measurements of fungal mycelium size were taken in 2-day intervals in the direction towards the embryo and in the direction opposite from the embryo. Control dishes were measured on four peripheral points corresponding to two perpendicular diameter axes. Each Petri dish was replicated four times.

Statistical analysis. The experiment follows $2 \times 3$ factorial design with replications. The objective was to describe growth of fungal tester reflecting hypothesized difference in defense reactions between the control (absent embryo) and two experimental variants (zygotic and somatic embryo) and also between the sides of measurement (towards and opposite from embryo) as a function of time from zero to ten days post inoculation.

An early inspection of mycelium size from control and experimental cultures suggested a sigmoid growth process, characterized by three-parametric logistic formula with a single upper asymptote, assumed by Koch (1975) for mycelial growth

$$
Y=\frac{\alpha}{1+e^{\left(\left(\beta_{0}-X\right) / \beta_{1}\right)}}+\varepsilon .
$$

In this equation, $Y$ was observed size of mycelium [mm], $\alpha$ was the coefficient of upper asymptote of the logistic curve, $\beta_{0}$ was the coefficient representing time required to reach inflection point positioned approximately at $\alpha / 2, \beta$ was the scale parameter indicating time required for mycelium to grow from half to $1 /\left(1+\mathrm{e}^{-1}\right) \approx 3 / 4$ of the asymptotic height, $\mathrm{X}$ was cultivation time of mycelium after inoculation in days and $\varepsilon$ represented random residual disturbances. Interpretations of the logistic parameters are illustrated in Fig. 1.

It is hypothesized, that model coefficients, primarily $\alpha$, are likely to vary, depending on all or some of the sources of variation, including two-way

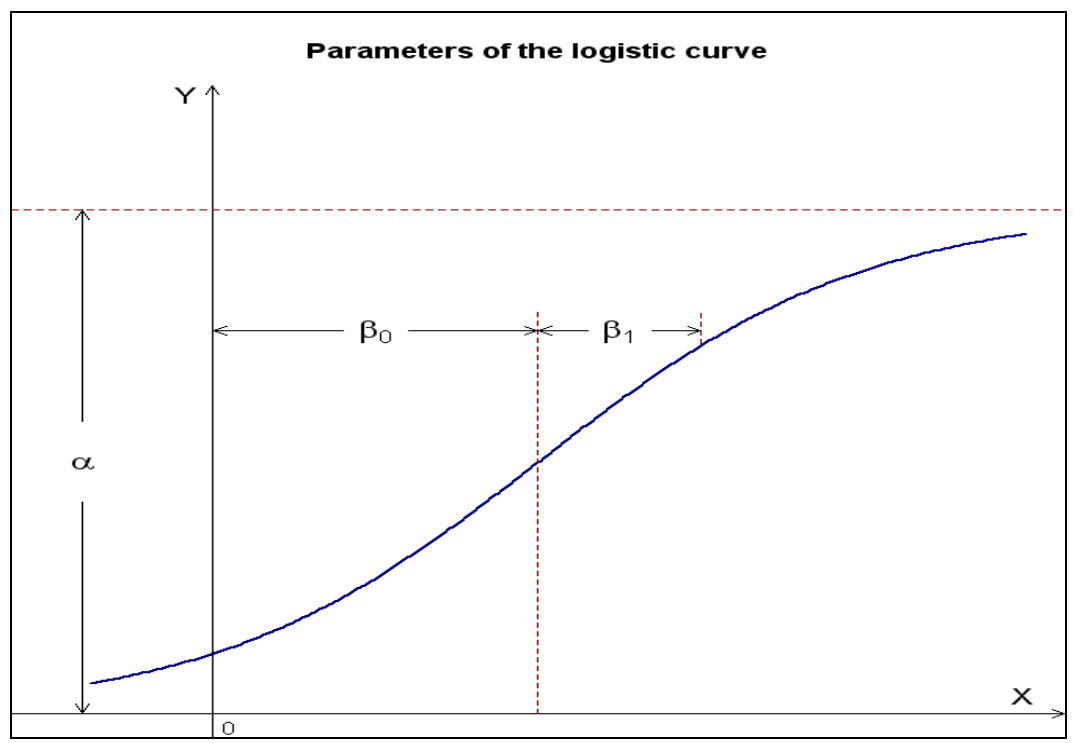

1 : Interpretation of the logistic curve parameters 
interactions. Model coefficients, especially $\alpha$ and to a smaller extent $\beta_{0}$ and $\beta_{1}$ are assumed to display noticeable inhibitory effects of plant embryos on mycelium growth. Several generalized fixed and mixed models were, as a result, constructed to reveal extent of the growth inhibition in response to embryo type (or control) and side of measurement. A general logistic model was constructed early for the combined data. In models with grouping factor, the model coefficients were allowed to fluctuate. Competing nested models were compared by likelihood ratio $\chi^{2}$ test to ascertain simultaneous significance of the added effects. Including random effects of Petri dishes on the logistic parameters was aimed at validating random variation associated with the dishes apart from the error term. Solutions to the coefficients $\alpha, \beta_{0}$ and $\beta_{1}$ were obtained by iterative Gauss-Newton algorithm. The process of iteration to convergence was traced.

Estimated parameterizations of the coefficients applied by statistical software due to issues of identifiability, were linearly transformed to improve interpretations and facilitate meaningful comparisons of fixed effects. Linear combinations were estimated using $\beta^{*}=C^{\prime} \beta$, where $C$ is a matrix storing coefficients of linear parametric functions and $\beta$ is vector of the non-transformed coefficients. Covariance matrix of the linear combinations was obtained by applying $\operatorname{Var}\left(\beta^{*}\right)=C^{\prime} \operatorname{Var}(\beta) C$, where $\operatorname{Var}(\beta)$ denotes the covariance matrix of the primary coefficients. Corresponding standard errors were received by taking square root of the matching main diagonal elements of the transformed parameter covariance matrix. Statistical significance of the coefficients and parametric functions was verified by regular t-test.

Mathematical properties of the transformed curves were assessed by finding root of the equation acquired by taking second derivative of the model with respect to time and setting equal to zero. Maximum gradient at the derived point of inflection was computed by maximizing the model first derivative with respect to time. Statistical analyses and construction of plots were accomplished with $\mathrm{R}$ software (www.r-project.org), particularly the built-in nlme library (version 3.1-95) and lattice library (version 0.19-23).

\section{RESULTS}

Coefficients of logistic growth curves in control (pure fungal tester) and experimental variants (zygotic or somatic embryo) were estimated by iteration after reaching convergence with the criterion $10^{-6}$. The logistic parameters estimated from the combined data disregarding the experimental grouping (Tab. I), were statistically significant $(p<0.01)$.

I: Estimated coefficients and associated $95 \%$ confidence limits from the general model

\begin{tabular}{lccc}
\hline Parameter & Estimate & $\mathbf{2 . 5 \%}$ & $\mathbf{9 7 . 5 \%}$ \\
\hline$\alpha$ & 30.77 & 29.14 & 32.40 \\
$\beta_{0}$ & 4.43 & 4.12 & 4.73 \\
$\beta_{1}$ & 1.51 & 1.27 & 1.76 \\
$\sigma_{e}$ & 3.57 & 3.20 & 4.04 \\
\hline
\end{tabular}

$\mathrm{f}(\mathrm{x})$ at point of inflection $=15.39 \mathrm{~mm}$; maximum gradient $=5.08 \mathrm{~mm} \cdot$ day $^{-1}$

To evaluate the defense reactions, fixed factors of embryo type and measurement sides towards or opposite from embryo and analogous twoway interactions were sequentially added to the model. The added fixed effects explained sufficient variation in the logistic growth, as confirmed by simultaneous likelihood-ratio test of the extra coefficients $\left(\chi^{2}=271.9, p<0.01\right)$ relative to the general unstructured model. Statistical significance of random effects associated with Petri dishes was not confirmed by the same test.

Linearly reparametrized coefficients for embryo types and control and measurement side combinations and respective standard errors were inferred from the original model (Tab. II). Estimated values of the growth curve at the inflection point $\left(\beta_{0}\right)$ reach approximately $50 \%$ of the asymptote $(\alpha)$, due to implicit symmetry in the logistic model. Maximum gradient at the inflection point indicate that the most rapid growth in the acceleration segment of the curve occurred in measurements on the opposite side from zygotic and somatic embryos. Variants towards the embryos, on the other hand, displayed the largest inhibition of growth, which

II: Estimated parameters of logistic curves, response at inflection point and maximum gradient at inflection point for embryo type and measurement side combinations

\begin{tabular}{lccccc}
\hline \multicolumn{1}{c}{ Combination } & $\alpha$ & $\beta_{0}$ & $\beta_{1}$ & $\begin{array}{c}\mathbf{f}(\mathbf{x}) \text { at point of } \\
\text { inflection [mm] }\end{array}$ & $\begin{array}{c}\text { Maximum gradient of } \\
\text { growth at point of inflection } \\
{\left[\mathbf{m m}_{\mathbf{d a y}} \mathbf{H}^{-1}\right]}\end{array}$ \\
\hline Control Opposite & $37.50^{* *}$ & $4.77^{* *}$ & $1.63 * *$ & 18.64 & 5.77 \\
Control Towards & $35.84^{* *}$ & $5.05^{* *}$ & $1.57^{* *}$ & 17.94 & 5.70 \\
Somatic Opposite & $32.70^{* *}$ & $4.02^{* *}$ & $1.36^{* *}$ & 16.26 & 6.03 \\
Somatic Towards & $27.80^{* *}$ & $4.84 * *$ & $1.75^{* *}$ & 13.93 & 3.96 \\
Zygotic Opposite & $29.34^{* *}$ & $3.68^{* *}$ & $1.19^{* *}$ & 14.79 & 6.18 \\
Zygotic Towards & $24.06^{* *}$ & $4.74 * *$ & $1.79^{* *}$ & 12.05 & 3.36 \\
\hline
\end{tabular}

Notation: ${ }^{* *}$ coefficients were significant at $\alpha=0.01$ 


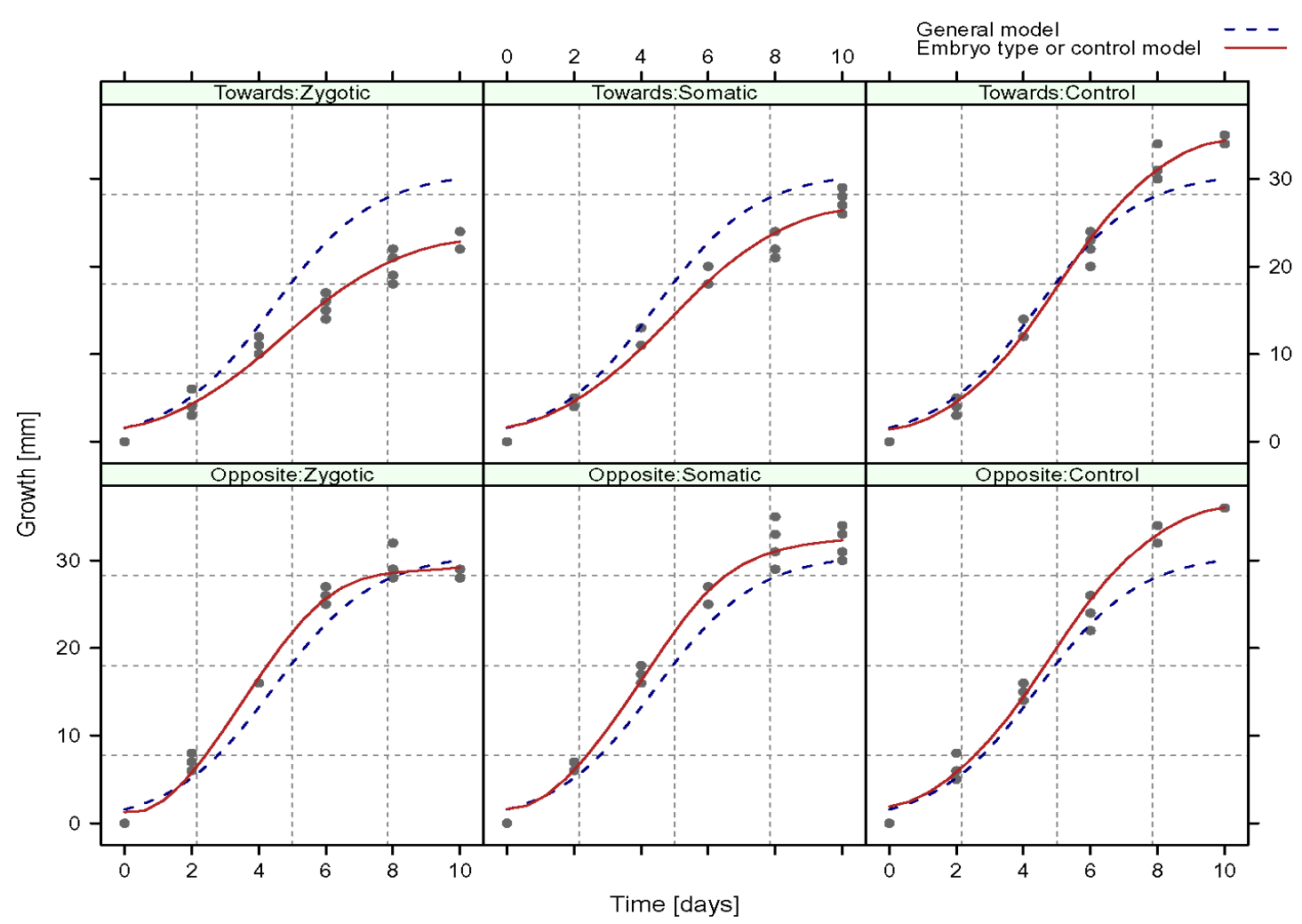

2: Estimated logistic curves for combinations of experimental variants and measurement side levels in the embryo type or control model and the ungrouped general model

was evident especially in growth towards the zygotic embryos. In the experimental variants, speed of growth opposite from the embryo also reached increased levels, compared to the control. This observation could be explained by elevated concentrations of the substances (auxin, lectins and others) in the cultivation media in the experimental dishes, where secondary metabolites produced by the embryo suppressed growth of fungal tester towards the embryo. Comparisons of the curves from the fixed effects model and from the general ungrouped model are provided in Fig. 2.

There is evidence, that presence of the embryo, embryo type (zygotic or somatic) and side of the measurement (towards or opposite from embryo) exert influence on the mycelium growth. Especially, the parameter $\alpha$ determining vertical size of the curve was strongly affected. Estimated coefficients for the combinations of embryo types or control and sides of measurement allow for construction of linear functions of the logistic parameters and statistical tests of their significance. Impact of the averaged effects of measurement sides and embryo types on all logistic coefficients is illustrated in Tab. III. Opposite variants had on average significantly higher upper asymptotes, required fewer days to reach the inflection point and needed less time to reach $3 / 4$ of the asymptote. Augmented speed of mycelium growth in the direction opposite from embryo was obvious in both embryo types.

Relative to control, the presence of embryos significantly reduced size of the logistic asymptote, especially when zygotic embryo was added, with reduction by almost $10 \mathrm{~mm}$, while it only slightly increased $\beta_{0}$ parameter having impact on duration of the acceleration phase (Fig. 3). Marginal effects of the embryo placement on $\beta_{1}$ coefficient, however, were not substantiated. Differences in vertical magnitude

III: Tests of main effects for measurement sides and experimental variants performed for parameters of logistic curves

\begin{tabular}{lcccc}
\hline \multirow{2}{*}{ Factor } & Main effects & \multicolumn{3}{c}{ Estimated parametric difference } \\
\cline { 3 - 5 } Sides & Opposite - Towards & $3.95^{* *}$ & $\beta_{0}$ & $-0.32 * *$ \\
& Control - Zygotic & $9.97 * *$ & $-0.72^{* *}$ & $0.11 \mathrm{~ns}$ \\
Experimental variants & Control - Somatic & $6.42^{* *}$ & $0.48^{* *}$ & $0.04 \mathrm{~ns}$ \\
& Somatic - Zygotic & $3.55^{* *}$ & $0.22 \mathrm{~ns}$ & $0.06 \mathrm{~ns}$ \\
\hline
\end{tabular}

Notation: differences were significant at $* * \alpha=0.01, * \alpha=0.05, \mathrm{~ns}=$ not significant 


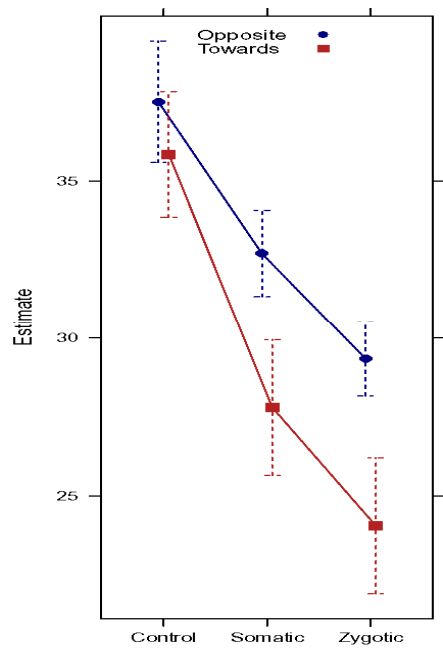

$\beta_{0}$

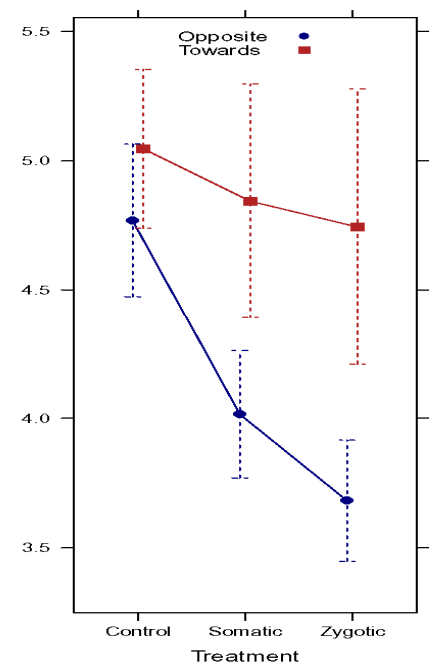

$\beta_{1}$

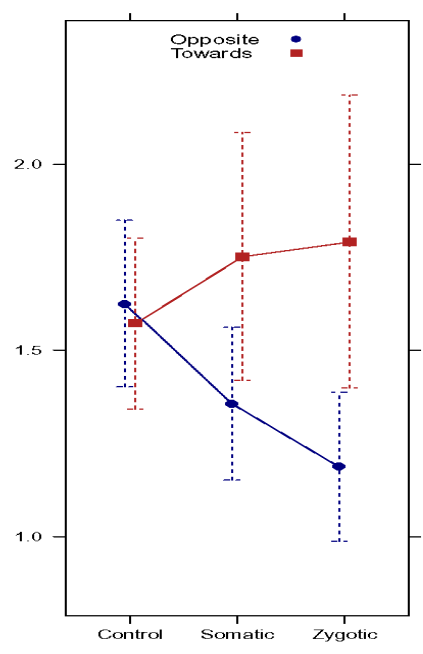

3: Estimated logistic coefficients and respective $95 \%$ confidence intervals for embryo type and measurement side combinations

IV: Tests of simple effects for experimental variants and measurement side factors holding the other factor fixed, for parameters of the logistic curve

\begin{tabular}{lccc}
\hline \multicolumn{1}{c}{ Simple effects } & \multicolumn{2}{c}{ Estimated parametric difference } \\
\cline { 2 - 4 } & $\alpha$ & $\beta_{0}$ & $\beta_{1}$ \\
\hline Control Opposite - Control Towards & $1.67 \mathrm{~ns}$ & $-0.28 \mathrm{~ns}$ & $0.05 \mathrm{~ns}$ \\
Somatic Opposite - Somatic Towards & $4.90^{* *}$ & $-0.83 * *$ & $-0.40^{*}$ \\
Zygotic Opposite - Zygotic Towards & $5.29 * *$ & $-1.06 * *$ & $-0.60 * *$ \\
Control Opposite - Somatic Opposite & $4.81^{* *}$ & $0.75 * *$ & $0.27 \mathrm{~ns}$ \\
Control Opposite - Zygotic Opposite & $8.16^{* *}$ & $1.09 * *$ & $0.44 * *$ \\
Somatic Opposite - Zygotic Opposite & $3.35^{* *}$ & $0.33 \mathrm{~ns}$ & $0.17 \mathrm{~ns}$ \\
Control Towards - Somatic Towards & $8.04 * *$ & $0.20 \mathrm{~ns}$ & $-0.18 \mathrm{~ns}$ \\
Control Towards - Zygotic Towards & $11.78^{* *}$ & $0.30 \mathrm{~ns}$ & $-0.22 \mathrm{~ns}$ \\
Somatic Towards - Zygotic Towards & $3.74 *$ & $0.10 \mathrm{~ns}$ & $-0.04 \mathrm{~ns}$ \\
\hline
\end{tabular}

Notation: differences were significant at ${ }^{* *} \alpha=0.01,{ }^{*} \alpha=0.05$, ns $=$ not significant

of growth curves ( $\alpha$ parameter) were also significant between somatic and zygotic embryo types.

Tests of simple effects are presented in Tab. IV. Relative to control, the presence of embryo still exerts decelerating influence on mycelium growth in the direction opposite from embryo $\left(\beta_{0}\right)$, as well as on the vertical size of the growth curve $(\alpha)$. The embryo appears to inhibit the growth on the side of the embryo, especially in late stages of the growth experiment, which is reflected by significant increase of $\beta_{1}$ and indicates slower growth in respective dishes. The restrictive effects of embryo placement on mycelium growth were also evident in the direction opposite from embryo, but to a lesser extent. This observation could be explained by release of substances inhibiting mycelium growth by the plant embryo and successive spread of the inhibitors by diffusion through the cultivation medium.

\section{DISCUSSION}

In previous study by Vooková et al. (2006), the defense reactions were apparent at very early stages of development in somatic embryos of A. numidica. Both embryonal suspensor mass (ESM) and early somatic embryos inhibited mycelial growth of tester P. schweinitzii. High endogenous levels of indole-3acetic acid (IAA) were confirmed by Michalczuk et al. (1992) in somatic embryos at initial stages of embryo development.

In present study, mature zygotic and desiccated somatic embryos of A. numidica were compared with respect to defense reactions. Fungal mycelium of P. schweinitzii in presence of embryos detected strong defense reactions manifested by slow growth of the fungus in comparison to control dishes. The differences in extent of defense reactions were described by logistic function and confirmed by statistical tests. Inhibition of fungal growth approximately 10 to $15 \%$ was observed in zygotic embryos relative to somatic ones. 
Etienne et al. (1993) measured contents of IAA and abscisic acid (ABA) in zygotic and somatic embryos and in the surrounding endosperm in Hevea brasiliensis. IAA and ABA contents in somatic embryos relative to zygotic ones were low and varied during ontogenesis. Woodward and Pearce (1988) studied inhibition of mycelial growth of P. schweinitzii. In general, increasing auxin concentration boosted the inhibition of fungal growth in presence of Sitka spruce callus. Mycelium of $P$. schweinitzii responded from concentration $10^{-6} \mathrm{~mol} \cdot \mathrm{l}^{-1}$ IAA in agar medium with inhibition of mycelial growth. At concentration $10^{-3} \mathrm{~mol} \cdot \mathrm{l}^{-1} \mathrm{IAA}$, a total growth inhibition was observed by Hřib et al. (1999). From the above compounds, auxin is ranked among the most effective regulators of plant growth. At concentrations above $10^{-2} \mathrm{~mol} \cdot \mathrm{l}^{-1}$, it is responsible for correlative inhibition of plant organs (Rittich et al., 1992). The results of current experiment suggest that fungus tester is sensitive to auxin.

In future experiments, we will attempt to confirm the idea of functional IAA mechanism in initial developmental phase of defense in conifer plant embryos by study of cationic isoperoxidases activity of both zygotic and somatic embryos of $A$. numidica.

\section{CONCLUSIONS}

In vitro cultivation of fungal tester in presence of mature zygotic and desiccated cotyledonary somatic embryos of A. numidica detected strong defense reactions manifested by inhibited growth of the tester compared to control dishes. The differences in extent of defense reactions in dual culture were described by logistic growth model and confirmed by statistical tests. Differences in the defense reactions were prominent especially in advanced stages of the co-cultivation. Exact biochemical processes behind the observed defense reactions remain yet to be explained. The fungal tester of P. schweinitzii, used in this study, could be applicable in future cultivation experiments with conifers.

In continuation of this experiment, it is possible to test defense reactions in desiccated cotyledonary somatic embryos from embryogenic cell lines of a particular coniferous plant species such as Abies alba or Pinus sylvestris. Eventually, comparison of defense reactions in zygotic embryos obtained from conifers of diverse origin can be proposed.

\section{SUMMARY}

Zygotic embryos of A. numidica were extirpated from the seeds. Somatic embryos were obtained from embryogenic tissue induced from immature zygotic embryos (Vooková and Kormuták, 2001). Defense reactions of desiccated cotyledonary somatic and mature zygotic embryos were tested by the technique of dual cultures with wood decaying fungus P. schweinitzii as tester. Growth response of Phaeolus schweinitzii mycelium to added embryos was studied on agar modified MS medium (B-25) without growth regulators. Inhibition in mycelium growth acted as a quantitative indicator of embryo defense reactions in somatic and zygotic embryo types, unrestricted control without embryo and sides of growth measurement (towards and opposite from embryo). The experiment follows $2 \times 3$ factorial design with replications. Mycelium growth was modeled by a logistic curve as a function of time from zero to ten days post inoculation. Differences in linearly transformed parameters of the curve were statistically tested in experimental and control variants and sides of measurement.

Experimental variants towards the embryo displayed the largest inhibition of growth, which was especially evident in impeded growth towards the zygotic embryo. Inhibitory reactions of somatic embryos were also significant, but only moderate in size. Standard errors of the parameters in the mycelium growth curves were slightly larger in embryo treated dishes. In conclusion, possible role of auxin (IAA) in manifestation of defense reactions is discussed, as a result of sensitivity of the tester towards auxin levels.

Dedication

The authors dedicate this article to commemorating the 100-th anniversary of birth of prof. RNDr. Vladimír Rypáček, DrSc.

\section{REFERENCES}

ETIENNE, H., SOTTA, B., MONTORO, P., MIGINIAC, E. and CARRON, M. P., 1993: Comparison of endogenous ABA and IAA contents in somatic and zygotic embryos of Hevea brasiliensis (Müll. Arg.) during ontogenesis. Plant Science, 92: 111-119.

HŘIB, J. and RYPÁČEK, V., 1981: A simple callus test to determine the aggressiveness of wood- 
destroying fungi. European Journal of Forest Pathology, 11: 270-274.

HŘIB, J., VOOKOVÁ, B. and FL'AK, P., 1999: In vitro growth response of wood-rotting fungi to IAA. Archiv für Phytopathologie und Pflanzenschutz, 32: 503-520.

HŘIB, J., VOOKOVÁ, B. and JANISCH, R., 1995ª: In vitro study of defense reaction of pine embryo and megagametophyte. Archiv für Phytopathologie und Pflanzenschutz, 529: 343-348.

HŘIB, J., VOOKOVÁ, B., SALAJOVÁ, T. BOLVANSKÝ, M. and FLAK, P., 1995' Testing of embryogenic and non-embryogenic calli of European black pine (Pinus nigra Arn.) for defense reactions to the fungus Phaeolus schweinitzii. Biologia, 50: 403-410.

KOCH, A. L., 1975: The kinetics of mycelial growth, Journal of General Microbiology, 89, 209-216.

MICHALCZUK, L., COOKE, T. J. and COHEN, J. D., 1992: Auxin levels at different stages of carrot somatic embryogenesis. Phytochemistry, 31: 1097-1103.

MURASHIGE, T. and SKOOG, F., 1962: A revised medium for rapid growth and bioassays with tobacco tissue cultures. Physiologia Plantarum, 15: 473-497.

OSTRY, M. E. and SKILLING, D. D., 1992: Application of tissue culture for studying tree defense mechanisms. In: Blanchette, R. A., Biggs, A. R. (eds.) Defense mechanisms of woody plants against fungi. Springer Verlag, New York, 405-423.
RITTICH, B., PIROCHTOVÁ, M., HŘIB, J., JURTÍKOVÁ, K. and DOLEŽAL, P., 1992: The antifungal activity of some aliphatic and aromatic acids. Collection of Czechoslovak Chemical Communications, 57: 1134-1142.

SCHENK, R. U. and HILDEBRANDT, A. C., 1972: Medium and techniques for induction and growth of monocotyledonous and dicotyledonous plant cell cultures. Canadian Journal of Botany, 50: 199_ 204.

TERHO,M.,PAPPINEN,A. and von WEISSENBERG, K., 2000: Growth reactions of a Gremmeniella abietina isolate and Scots pine embryogenic tissue cultures differ in a host-parasite in vitro system. Forest Pathology, 30(5): 285-295.

VOOKOVÁ, B. and KORMUŤÁK, A., 200l: Effect of sucrose concentration, charcoal, and indole3-butyric acid on germination of Abies numidica somatic embryos. Biologia Plantarum, 44: 181184.

VOOKOVÁ, B., KORMUŤÁK, A. and HŘIB, J., 2001: Effect of myo-inositol on somatic embryogenesis of Abies numidica. Journal of Applied Botany, 75: 46-49.

VOOKOVÁ, B., HŘIB, J., KORMUŤÁK, A. and ADAMEC, V., 2006: Defense reactions of developing somatic embryos of Algerian fir (Abies numidica). Forest Pathology, 36: 215-224.

WOODWARD, S. and PEARCE, R. B., 1988: Responses of Sitka spruce callus to challenge with wood decay fungi. European Journal of Forest Pathology, 18: 217-229.

Address

Ing. Jiří Hřib, CSc., Dr.h.c., Ukrajinská 17, 62500 Brno, Česká republika, doc. Ing. Václav Adamec, Ph.D., Ústav statistiky a operačního výzkumu, Mendelova univerzita v Brně, Zemědělská 1, 61300 Brno, Česká republika, RNDr. Božena Vooková, CSc., Ústav genetiky a biotechnológií rastlín, Akademie vied SR, Akademická 2, P.O. BOX 39A, 95007 Nitra, Slovenská republika, e-mail: jhribl@nbox.cz, vadamec@mendelu.cz, nrgrvook@ savba.sk 
